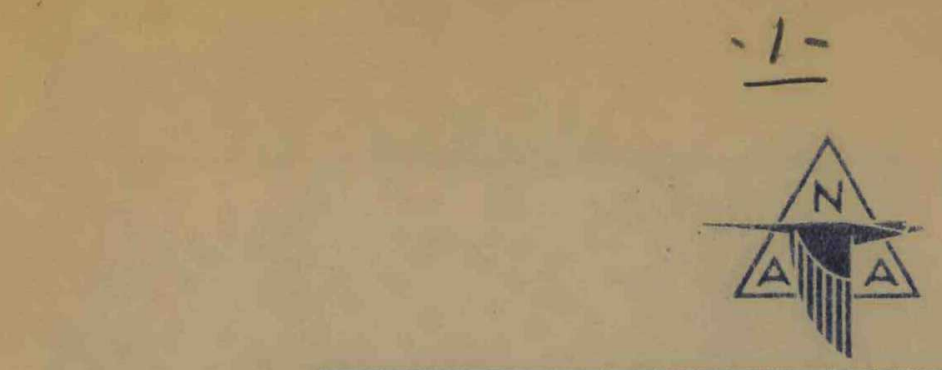

\title{
A VAPOR PRESSURE CHART FOR METALS
}

This document is PUBLICLY RELASA

A. Kivs Authorizing Oiich Date:

\section{BY:}

\section{R. L. LOFTNESS}

This report has been photostated to fill your request as our supply of copies was exhausted. If you should find that you do not need to retain this copy permanently in your files, we would greatly appreciate your returning it to TIS so that it may be used to fill future requests from other AEC installations.

H. PEARLMAN, GROUP LEADER-CHEMISTRY

\section{ATOMIC ENERGY RESEARCH DEPARTMENT NORTH AMERICAN AVIATION, INC. \\ P. O. BOX 309




\section{DISCLAIMER}

Portions of this document may be illegible in electronic image products. Images are produced from the best available original document. 


\section{ABSTRACT}

The plot of $\log \mathrm{p}-\mathrm{ZS}-1 / \mathrm{T}$ data for 52 metallic or refractory elements yields a family of stralght lines which converge within a small region near 10,000 atmospheres and $10,000^{\circ} \mathrm{C}$. (This is similar to the Cox chart for organic 11quids.)

Establishing an approximate convergence point permits useful estimation of the entire $\log \mathrm{p}$ ys $\frac{1}{\mathrm{~T}}$ line up to $10,0000^{\mathrm{C}}$. for a metal, provided a single value of the vapor pressure is known at any temperature. Only $\mathrm{Zn}, \mathrm{Cd}$, Hg and Cs deviate substantially from the convergent line pattern of all the elements studied.

This report is besed on studies conducted for the Atomic Energy Commission under Contract AT-11-1-GEN-8.

This document is PUBLICLY RELTASABLE

At Kaise

Authorizing Oincia

Date: $10-11-11$ 


\section{3}

\section{DISCUSSION}

The Clausius-Clapeyron equation represents the change in equilibrium pressure with the temperature for any phase equilibrium in a single-component system.

$$
\frac{d p}{d T}=\frac{\lambda}{T \Delta v}
$$

where $\lambda=$ latent heat of phase change

$$
\Delta=\text { volume change accompanying the phase change. }
$$

Neglecting the volume of Ilquid in comparison with that of the vapor and assuming that the vapor is an ideal gas, Eq. (1) becomes,

$$
\frac{\mathrm{d} \ln \mathrm{p}}{\mathrm{dT}}=\frac{\lambda}{R \mathrm{~T}^{2}}
$$

Assuming that $\lambda$ is Independent of temperature and integrating we have

$$
\ln p=\frac{-\lambda}{R T}+\text { Constant }
$$

or as an empirical equation

$$
\log p=-\frac{A}{T}+B
$$

By plotting the experimental data given in Table I it becomes apparent that the vapor pressure curves converge within a small area. By picking the center of this area as a point of convergence it is possible to construct a chart such as Fig. I which gives straight line values for the vapor pressure of the metals as a function of temperature. The point of convergence is 


\section{4}

roughly at 10,000 atmospheres and $10,000^{\circ} \mathrm{C}$.

Not all metals have vapor pressure-temperature curves of the proper slope to converge at the selected point although the divergence is not more than $\pm 50^{\circ} \mathrm{C}$. for a given vapor pressure on a line drawn through the convergence point and the boiling point of the metal. The metals which deviate the most from the slope predicted by the chart are $\mathrm{Zn}, \mathrm{Cd}, \mathrm{Hg}$, and Cs. It will be noted that these are anong the most volatile of all the elements listed.

It is possible from $\mathrm{FIg} .1$ to estimate vapor pressure at any temperature for a metal where only one vapor pressure value is known. It should be noted that the values of the boiling points listed in Ref. 2 for the more refractory metals are in many cases too high according to the chart (assuming that the low pressure data is more accurate than the determination of the boiling points). Al, Ga, Ge ${ }_{n}^{A N O}$ In have recorded boiling points about $1000^{\circ} \mathrm{C}$. too high; $\mathrm{Th}, \mathrm{Nb}, \mathrm{Sn}$, Mo, and $\mathrm{W} 300$ to $500^{\circ} \mathrm{C}$. too high; and Ta has a recorded bolling point which is $1100^{\circ} \mathrm{C}$. too high according to the chart. However, the temperature scale becomes very condensed at higher temperatures (1200 to $10,000 \circ \mathrm{C}$ ) and the accuracy to be expected in plotting and readine temperatures is \pm 50 to $100^{\circ} \mathrm{C}$.

The constants $A$ and B in Eq. (4) determined from Fig. I are given in Table II. For comparison, the same constants are listed as given in the literature. The values of A (representing the slope of the vapor pressure curve) taken from Fig. 1 and from the literature are in good agreement 


\section{5}

except for $\mathrm{Ba}$ and $\mathrm{Sb}$. The values of $\mathrm{B}$, representing the intercept of the vapor pressure curve at $I / T=0$, do not agree as closely.

\section{CONCLUSIONS}

Although the observed vapor pressure data for a few metals does not fit exactly the values predicted in Fig. I there is a close enough correlation for most metals to give the chart some utility in estimating vapor pressures of metals. 
1. Dushman, S., Scientific Foundation of Vacuum Techniaue, John Wiley and Sons, Inc., New York. Chap. 11, 1949

2. Lyon, R. N., Liquid Metals Handbook, U. S. Coverment Printing Office, 1950. p. 31.

3. Stull, D. R., "Vapor Pressure of Pure Substances," Ind. and Eng. Chem,, 39, $517(1947)$.

4. Sinithells, C. J., Metals Reference Book, Interscience Publishers, New York, 1949. p. 249. 
TABLE I

VAPOR PRESSURES OF METALS

\begin{tabular}{|c|c|c|c|c|c|c|c|c|c|}
\hline \multirow[t]{2}{*}{ Metal } & \multicolumn{6}{|c|}{$\begin{array}{l}\text { Temperatures (In }{ }^{\circ} \text { ) at Which the } \\
\text { Vapor Pressures are }\end{array}$} & \multirow{2}{*}{$\begin{array}{l}\text { Bolling } \\
\text { Point }{ }^{\circ} \mathrm{C} \\
760 \text { (a) } \\
\text { (a) }\end{array}$} & \multirow{2}{*}{$\begin{array}{l}\text { Boiling } \\
\text { Point }{ }^{\circ} \\
\text { (From } \\
\text { fic. I) } \\
\end{array}$} & \multirow{2}{*}{$\begin{array}{c}\text { Melting } \\
\text { Point } \\
{ }^{\circ} \\
\text { (a) }\end{array}$} \\
\hline & $\begin{array}{r}0.001 \mathrm{~mm} \\
\text { (a) }\end{array}$ & $\begin{array}{r}0.01 \mathrm{~mm} \\
(\mathrm{a})\end{array}$ & $\begin{array}{l}0.1 \mathrm{~mm} \\
\text { (a) }\end{array}$ & $\begin{array}{r}1.0 \mathrm{~mm} \\
(\mathrm{a})\end{array}$ & $\begin{array}{r}10 \mathrm{~mm} \\
\text { (a) }\end{array}$ & $\begin{array}{r}100 \mathrm{~mm} \\
\text { (a) }\end{array}$ & & & \\
\hline LI & 439 & 514 & 607 & 725 & 890 & 1084 & 1367 & 1330 & 179 \\
\hline $\mathrm{Na}$ & 238 & 291 & 356 & 437 & 548 & 696 & 914 & 880 & 98 \\
\hline $\mathrm{K}$ & 161 & 207 & 265 & 338 & 443 & 581 & 779 & 730 & 64 \\
\hline $\mathrm{Rb}$ & 123 & 165 & 217 & 283 & 387 & 519 & 679 & 640 & 38.5 \\
\hline Cs & 110 & 153 & 207 & 277 & 387 & 515 & 690 & & 29 \\
\hline $\mathrm{Cu}$ & 1141 & 1273 & 1432 & 1628 & 1879 (c) & 2207 (c) & 2595 & 2610 & 1083 \\
\hline $\mathrm{Ag}$ & 936 & 1047 & 1184 & 1353 & 1575 (c) & $1865(\mathrm{c})$ & 2212 & 2240 & 961 \\
\hline $\mathrm{Au}$ & 1316 & 1465 & 1646 & 1867 & 2154 (c) & 2521 (c) & 2966 & 2900 & 1063 \\
\hline $\mathrm{Be}$ & 1130 & 1246 & 1395 & 1582 & & & 2507 & 2550 & 1284 \\
\hline $\mathrm{Mg}$ & 383 & 443 & 515 & 605 & 702 & 909 & 1126 & 1130 & 651 \\
\hline $\mathrm{Ca}$ & 528 & 605 & 700 & 817 & 983 (c) & 1207 (c) & 1482 & 1480 & 810 \\
\hline Sr & 475 & 549 & 639 & 750 & 898 (c) & 1211 (c) & 1384 & 1380 & $7 n$ \\
\hline $\mathrm{Ba}$ & 625 & 721 & 840 & 961 & 1049 (c) & 1301 (c) & 1638 & 1670 & 717 \\
\hline $\mathrm{Zn}$ & 292 & 343 & 405 & 487 (b) & 593 & 736 & 907 & 940 & 419 \\
\hline $\mathrm{Cd}$ & 220 & 264 & 321 & 394 (b) & 484 & 611 & 765 & & 321 \\
\hline $\mathrm{Hg}$ & 18 & 48 & 82 & 126 & 184 & 261 & 361 & 360 & -40 \\
\hline$B^{\circ}$ & 1239 & 1355 & 1489 & 1648 & & & 2527 & 2700 & 2000 \\
\hline $\mathrm{A}]$ & 889 & 996 & 1123 & 1279 & 1487 & 1749 & 2327 & 2200 & 660 \\
\hline $\mathrm{Se}$ & 1282 & 1423 & 1595 & 1804 & & & 2727 & 2800 & 1397 \\
\hline$Y$ & 1494 & 1649 & 1833 & 2056 & & & 3227 & & 477 \\
\hline La & 1242 & 1381 & 1549 & 2754 & & & 2727 & & 887 \\
\hline $\mathrm{Ce}$ & 1190 & 1305 & 1439 & 1599 & & & 2527 & & 785 \\
\hline $\mathrm{Ga}$ & 965 & 1093 & 1248 & 1443 & 1541 (c) & 1784 (c) & 2427 & 2300 & 30 \\
\hline In & 840 & 952 & 1088 & 1260 & 14,66 & 1756 & 2167 & 2080 & 157 \\
\hline$M$ & 527 & 606 & 702 & 821 & 983 & 1196 & 1457 & 1450 & 304 \\
\hline c & 2471 & 2681 & 2926 & 3214 & $3946(c)$ & 4373 (c) & 4552 & 4500 & \\
\hline S1 & 1223 & 1343 & 1485 & 1670 & $1888(\mathrm{c})$ & 2083 (c) & 2477 & 2730 & $1 / 10$ \\
\hline $\mathrm{Ti}$ & 1384 & 1546 & 1742 & 1965 & & & 3127 & 3000 & 1727 \\
\hline $\mathrm{Zr}$ & 1816 & 2001 & 2212 & 2459 & & & 3577 & 3700 & 2127 \\
\hline $\mathrm{Th}$ & 1999 & 2196 & 2431 & 2715 & & & 4227 & 3900 & 1827 \\
\hline$G_{\theta}$ & 1112 & 1251 & 1421 & 1635 & & & 2707 & 2600 & 959 \\
\hline $\mathrm{Sn}$ & 1042 & 1189 & 1373 & 1609 & 1703 & 1968 & 2727 & 2330 & 232 \\
\hline $\mathrm{Pb}$ & 625 & 718 & 832 & 975 & 2167 & 1417 & 1737 & 1700 & 328 \\
\hline$\nabla$ & 1725 & 1888 & 2079 & $220 ?$ & & & 3527 & 3500 & 1697 \\
\hline $\mathrm{Cb}$ & 2539 & & & & & & 5127 & 4600 & 2500 \\
\hline $\mathrm{Ta}$ & 2820 & & & & & & 6027 & 4900 & 2996 \\
\hline $\mathrm{Sb}$ & 595 & 678 & 779 & 904 & 1033 & 1223 & 1617 & 1620 & 630 \\
\hline B1 & 609 & 698 & 802 & 934 & 1136 (e) & 1271 (c) & 1627 & 1650 & 271 \\
\hline Cr & 1090 & 1205 & 1342 & 1504 & & & 2222 & 2510 & 1900 \\
\hline Mo & 2295 & 2533 & & $3102(c)$ & 3535 (c) & 4109 (c) & 4804 & 4300 & 2622 \\
\hline $\mathrm{W}$ & 3016 & 3309 & & 3990 (c) & 4507 (c) & $5168(c)$ & 5677 & 5100 & 3382 \\
\hline U & 1730 & 1898 & 2098 & 2338 & & & 3527 & 3500 & 1132 \\
\hline $\mathrm{Mn}$ & 878 & 980 & 1103 & 1251 & 1505 (c) & $2792(\mathrm{c})$ & 2097 & 2130 & 1244 \\
\hline $\mathrm{Fe}$ & 1310 & 1447 & 1602 & 1783 & 2039 (c) & $2360(c)$ & 2727 & 2850 & 1535 \\
\hline Co & 1494 & 1649 & 1833 & 2056 & & & 3097 & 3260 & 1478 \\
\hline $\mathrm{NI}$ & 1371 & 1510 & 1679 & 1884 & 2507 (c) & 2364 (c) & 2837 & 3000 & 1455 \\
\hline Ru & 2230 & 2431 & 2666 & 2946 & & & 4227 & 4250 & 2427 \\
\hline $\mathrm{Rh}$ & 1971 & 2149 & 2358 & 2607 & & & $38 \pi 7$ & 3850 & 1967 \\
\hline $\mathrm{Pd}$ & 3405 & 1566 & 1759 & 2000 & & & 3167 & 3100 & 1555 \\
\hline$O_{s}$ & 2451 & 2667 & 2920 & 3221 & & & 4627 & 4500 & 2697 \\
\hline Ir & 2340 & 2556 & 2811 & 3118 & & & 4527 & 4400 & 2454 \\
\hline$P t$ & 1904 & 2090 & 2313 & 2582 & 3146 (c) & 3714 (c) & 3827 & 3800 & 1774 \\
\hline
\end{tabular}

a. From Ref. 1

b. From Ref. 2

c. From Ref. 3 
9 TAARLB II

CONSTANTS FOR VAPOR PRESSURE

EQUATION $\log p=\frac{A}{T}+B$

\begin{tabular}{|c|c|c|c|c|}
\hline Element & $\begin{array}{c}\text { A } \\
\text { From Fig. } 1\end{array}$ & $\begin{array}{c}\text { A } \\
\text { From Ref. } 4\end{array}$ & $\begin{array}{c}\text { B } \\
\text { From Fig. I }\end{array}$ & $\begin{array}{c}\text { B } \\
\text { From Ref. } 4\end{array}$ \\
\hline $\begin{array}{l}\mathrm{W} \\
\mathrm{Ta} \\
\mathrm{Nb} \\
\mathrm{C} \\
\mathrm{Og} \\
\mathrm{Ir} \\
\mathrm{Mo} \\
\mathrm{Ru} \\
\mathrm{Th} \\
\mathrm{Rh} \\
\mathrm{Pt} \\
\mathrm{Zr} \\
\mathrm{U} \\
\mathrm{V} \\
\mathrm{Co} \\
\mathrm{Pd} \\
\mathrm{TI} \\
\mathrm{Ni} \\
\mathrm{Au} \\
\mathrm{Fe} \\
\mathrm{Sc} \\
\mathrm{SI} \\
\mathrm{B} \\
\mathrm{Cu} \\
\mathrm{Ge} \\
\mathrm{Be} \\
\mathrm{Cr} \\
\mathrm{Sn} \\
\mathrm{Ga} \\
\mathrm{Ag} \\
\mathrm{AI} \\
\mathrm{Mn} \\
\mathrm{In} \\
\mathrm{Pb} \\
\mathrm{Ba} \\
\mathrm{BI} \\
\mathrm{Sb} \\
\mathrm{Ca} \\
\mathrm{TI} \\
\mathrm{Sr} \\
\mathrm{LI} \\
\mathrm{Mg} \\
\mathrm{Zn} \\
\mathrm{Na} \\
\mathrm{K} \\
\mathrm{Rb} \\
\mathrm{Hg}\end{array}$ & $\begin{array}{l}49300 \\
45100 \\
39200 \\
37700 \\
37000 \\
35400 \\
34700 \\
33300 \\
29400 \\
29100 \\
27900 \\
26300 \\
25000 \\
25000 \\
21400 \\
20000 \\
19700 \\
19700 \\
18900 \\
18700 \\
18200 \\
17900 \\
17200 \\
16600 \\
16300 \\
16000 \\
15700 \\
14400 \\
14000 \\
13700 \\
13300 \\
12900 \\
12600 \\
10000 \\
9900 \\
9600 \\
9500 \\
8650 \\
8450 \\
8100 \\
7750 \\
6680 \\
56400 \\
5370 \\
4570 \\
4250 \\
2760\end{array}$ & $\begin{array}{l}42,000 \\
39,800 \\
\\
\\
34,000 \\
\\
\\
27,900 \\
\\
\\
\\
\\
\\
12,100 \\
19,400 \\
19,300 \\
19,300 \\
18,000 \\
17,100 \\
17,700 \\
17,300 \\
17,800 \\
17,400 \\
16,300 \\
14.550 \\
16,450 \\
13,260 \\
12,200 \\
10,300 \\
350,000 \\
10,000 \\
614 \\
9,500 \\
9,300 \\
8,860 \\
7,610 \\
6,670 \\
5,700 \\
4,770 \\
4,280 \\
3,328\end{array}$ & $\begin{array}{l}11.8 \\
17.3 \\
11.0 \\
10.7 \\
10.7 \\
10.4 \\
10.3 \\
10.2 \\
10.0 \\
9.9 \\
9.7 \\
9.5 \\
9.34 \\
9.34 \\
9.15 \\
9.12 \\
9.00 \\
9.00 \\
8.82 \\
8.80 \\
8.70 \\
8.60 \\
8.60 \\
8.50 \\
8.45 \\
8.40 \\
8.37 \\
8.28 \\
8.26 \\
8.25 \\
8.23 \\
8.20 \\
8.18 \\
8.11 \\
8.00 \\
7.95 \\
7.95 \\
7.80 \\
7.75 \\
7.67 \\
7.65 \\
7.50 \\
7.38 \\
7.36 \\
7.30 \\
7.28 \\
7.20\end{array}$ & $\begin{array}{r}7.87 \\
12.50 \\
9.00 \\
12.83 \\
12.31 \\
13.51 \\
9.22 \\
10.37 \\
9.28 \\
14.12 \\
10.13 \\
12.36 \\
8.63 \\
8.03 \\
11.43 \\
15.77 \\
12.70 \\
-26.30 \\
11.91 \\
11.10 \\
12.50 \\
11.6 \\
12.0 \\
11.33 \\
11.6 \\
11.34 \\
10.5\end{array}$ \\
\hline
\end{tabular}




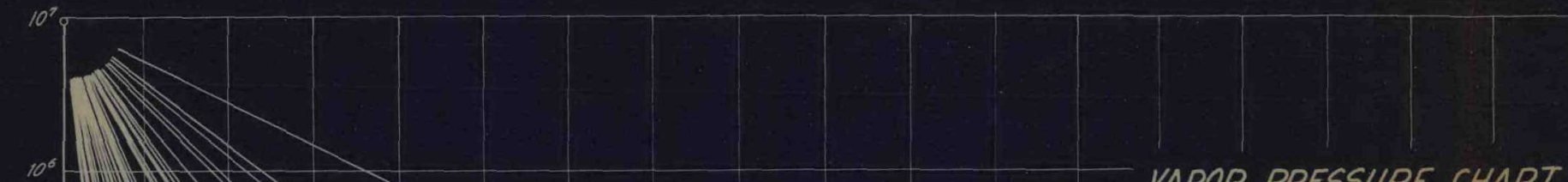

VAPOR PRESSURE CHART
FOR MEIALS

$\triangle=$ MELING POINTS

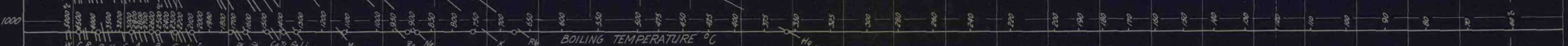

है

है
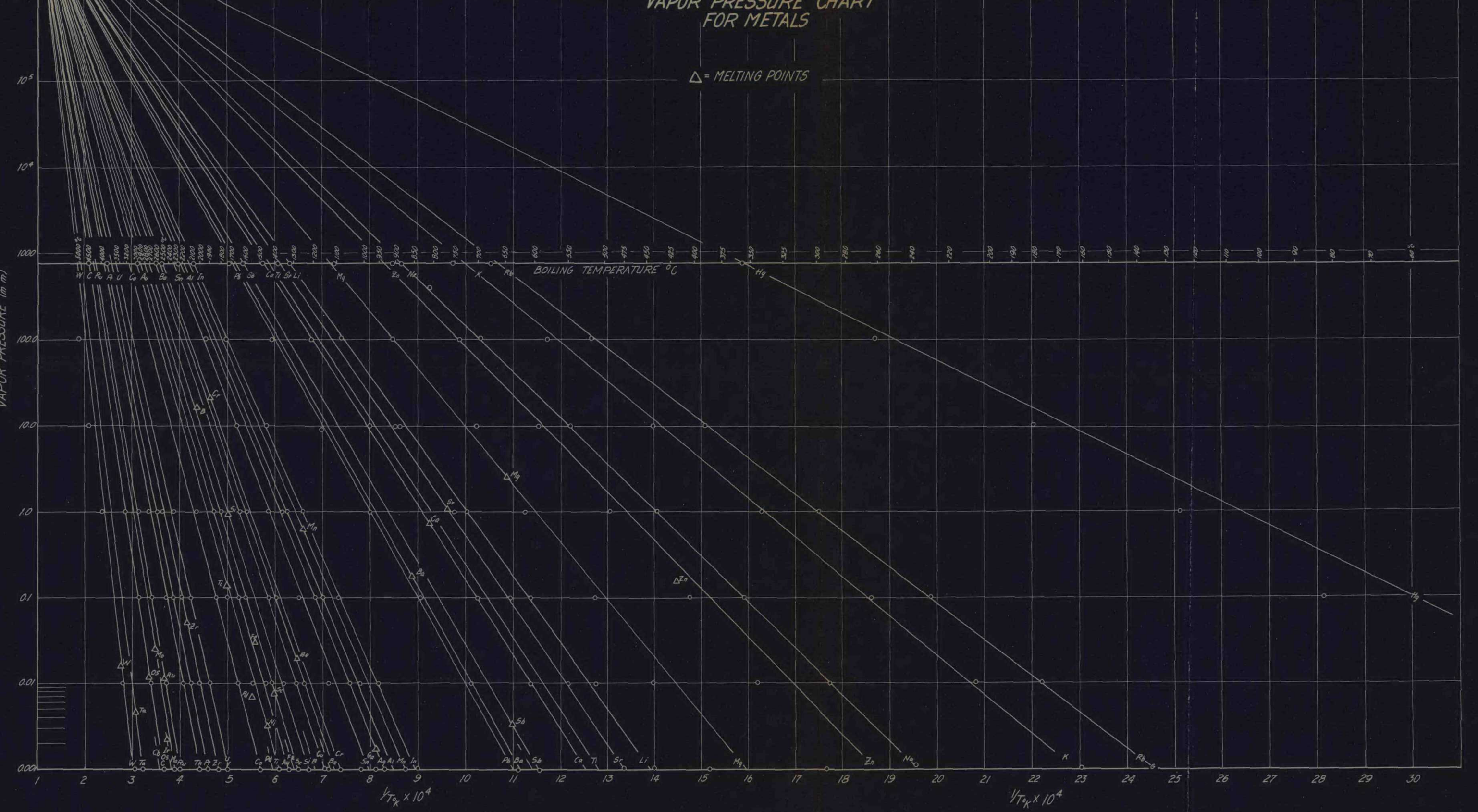

FI6URE 